\title{
Auditoria de Gestión como Instrumento en la Decisión Gerencial
}

\author{
Management Audit As An Instrument In The Management \\ Decision
}

\section{RESUMEN}

La existencia de la necesidad de información por los altos directivos en las entidades públicas o privadas para la toma de decisiones, las que adolecen muchas veces de pruebas objetivas independientes que pudieran aumentar su eficiencia, además los auditores, hoy orientan sus prácticas de auditoría hacia los estados financieros y/u operativos, lo realizan con un alcance menor que no les permite incluirlas dentro de su opinión o dictamen. El objetivo de la auditoría de gestión, tal como se plantea en el presente artículo, es el de una auditoria con evaluación en la gestión, toma de decisiones de la alta dirección, en el cumplimiento de normas y procedimientos, con un alcance suficiente que permita concluir u opinar sobre estos temas. Palabras claves: Decisiones; auditoria; gestión.

\begin{abstract}
The existence of the need for information by senior managers in public or private entities for decision making, which often suffer from independent objective tests that could increase their efficiency. The auditors today direct their auditing practices towards the financial and / or operational statements perform it with a smaller scope that does not allow them to include them in their opinion or dictum.

The objective of the management audit, as set out in this article, is an audit with evaluation in the management, decision making of senior management, in compliance with standards and procedures, with a sufficient scope to allow conclude or comment on these issues.
\end{abstract}

Keywords: Decisions; audit; management.

\section{Félix Armando Rivera León ${ }^{1}$}

felix_arl@hotmail.com

Félix A. Rivera Sandoval ${ }^{2}$

riverasandovalfelix@gmail.com

Universidad Nacional Mayor de San Marcos

\footnotetext{
1 Doctor en Ciencias Contables y Empresariales por la Universidad Nacional Mayor de San Marcos (UNMSM), Magister en Administración con mención en Gestión Empresarial (UNMSM), Contador Público Colegiado con 38 años de experiencia en la especialidad de Auditoria, Profesor Principal de la Facultad de Ciencias Administrativas (UNMSM), ha desempeñado diversos cargos en la Facultad y en entidades del sector público y privado.

2 Maestrista en Administración- Gestión Empresarial (UNMSM), Analista de Gestión Operativa - M y E Servicios Generales, Funcionario de Instituciones Públicas

(c) Los autores. Este artículo es publicado por Gestión en el Tercer Milenio de la Facultad de Ciencias Administrativas de la Universidad Nacional Mayor de San Marcos. Este es un artículo de acceso abierto, distribuido bajo los términos de la licencia Creative Commons Atribucion - No Comercia_Compartir Igual 4.0 Internacional. (http://creativecommons.org/licenses/by-nc-sa/4.0/) que permite el uso no comercial, distribución y reproducción en cualquier medio, siempre que la obra original sea debidamente citada.
} 


\section{INTRODUCCIÓN}

Las entidades organizacionales requieren perspectivas y herramientas que permitan estar a tono con la evolución de su entorno y de las necesidades del mercado incluso las técnicas que diagnostiquen e identifiquen los elementos internos y externos que no se alinean como deben técnicas que sugieran pautas de innovación de los elementos fallidos u obsoletos.

El presente artículo centra su discurso con el objetivo de dar respuesta a estos problemas y expectativas. Por ello se enfoca en lo denominado Auditoria de Gestión cuya misión es la evaluación, análisis e innovación en las entidades organizacionales.

Vista la organización bajo una visión integral, el entorno competitivo y las teorías de la organización, es posible proponer diagnósticos y proposiciones más intensas y realistas.

Desde el punto de vista metodológico, nuestro estudio tiene un enfoque de investigación de tipo Abductivo creativo, porque parte hechos explicados de tipo inductivo, que han permitido esclarecer una verdad (marco teórico); por tanto, considerada cierta, se debe lograr un nuevo caso explicativo y plausible, forma parte del cambio epistémico por medio de la cual se pretende nueva posición de los hechos; la explicación que se presenta debe ser simplemente una sugerencia que deberá ser puesta en prueba antes de ponerse como creencia teórica.

En la primera parte nos centramos en determinar los objetivos propios de las prácticas de auditoria a emplearse en los exámenes requeridos. Para luego establecer los alcances supeditadas al área examinada, identificando y analizando las causas de las ineficiencias e ineficacias y sus consecuencias.

El planteamiento de los estudios e investigaciones realizadas por los auditores deberán estar señalizadas dentro de las normas generalmente aceptadas, prestablecidos por instituciones tutelares de las profesiones y especialidades de auditoria internacionalmente; en ello nos centramos, describiendo algunas de ellas, competencia del presente artículo.

Para luego desembocar en la narración de la metodología adoptada, y posibles desarrollos de las propias prácticas de auditoria en búsqueda de objetivos planteados en los programas de trabajo previamente realizados.

Finalmente concluimos en afirmar que el contenido del presente artículo muestra el enfoque y planteamiento metodológico de cómo se debe llevar a cabo la práctica del examen. Reiteramos, la explicación que se presenta debe ser simplemente una sugerencia que deberá ser puesta en prueba antes de ponerse como creencia teórica.

\section{OBJETIVO}

Existe un gran número de revistas y libros publicados sobre Auditoria en sus diversos campos de aplicación, hasta ahora no existe uniformidad de criterios sobre que es la auditoria, cómo se lleva a cabo, quien la efectúa o cuales deben ser sus resultados.

El propósito del presente artículo no es presentar, discutir y evaluar, las diferentes filosofías, definiciones o metodologías propuestas sobre el tema, sino presentar una breve exposición de ideas, fundamentos, procesos y resultados en las prácticas de evaluación que el auditor deberá realizar frente al desempeñó gerencial, en opinión de autor.

\section{ARGUMENTACIÓN}

La Auditoría de Gestión ha evolucionado, de la Auditoria Financiera. Emplea las mismas técnicas de investigación y pruebas, y metodología muy similar aplicadas a los aspectos operativos y actividades realizadas en sus prácticas de trabajo.

Una gerencia eficiente es la que acepta el proceso de cambio. Es una gerencia que continuamente busca la mejora a través de la eficiencia, eficacia y economía. Es una gerencia que quiere eliminar sistemas y métodos antiguos ineficaces, operaciones poco productivas y actividades no significativas que desvían a la empresa del logro de su misión principal y de sus objetivos definidos. Es una gerencia que trata de conocer sus áreas críticas, sus puntos débiles y problemas más significativos. Debe ser una gerencia que depende del conocimiento oportuno de las acciones correctivas a tomarse y de la reforma a realizarse. A menudo la gerencia y los directivos de la empresa no logran una dinámica 
debido a que se encuentran involucrados en las operaciones diarias y no pueden darse el lujo de planear operaciones futuras. Pueden conocer en forma general los problemas, pero no en buscar sus soluciones. A menudo se encuentran tan involucrados que pierden la visión necesaria para mejorarlos.

Es aquí que la gerencia requiere de un instrumento que le proporcione una dinámica. Un instrumento que se dedique exclusivamente a la identificación de problemas, de la evaluación de riesgos, medición de sus efectos, identificación de las causas y recomendaciones que deben tomarse y mejorar las operaciones. Este instrumento es la Auditoria de Gestión.

Si bien es cierto de la existencia de varias terminologías en cuanto a conceptos de auditoría, ellas se refieren a los diferente enfoques de aplicación de acuerdo a los objetivos de la misma; ejemplo: Auditoria administrativa; auditoría Financiera; Auditoria de Procesos; Auditoria Operativa; Auditoria Operacional o de Actividades; Auditoria Integral, las que realizaran sus prácticas de auditoría, bajo las mismas técnicas y normatividades existentes profesionalmente, pero con diferentes objetivos y alcances de trabajo.

Bajo este contexto la Auditoria de Gestión se encuentra orientada a establecer los objetivos siguientes:

- Verificar el apropiado manejo de los recursos;

- Comprobar la existencia de métodos o procedimientos apropiados de operación y de eficiencia de los mismos;

- Verificar la confiabilidad de la información y de los controles pre establecidos;

- Vigilia de la existencia de políticas y el cumplimiento de las mismas;

- Comprobar la existencia de objetivos y planes adecuados y realistas;

- Evaluar lo adecuado de la organización de la institución o empresa.

Coherente con lo que postula: Dykerman (1969). Propone los siguientes objetivos fundamentales en una auditoria gerencial: a. "Detectar sectores en los que existe un peligro o perdida potenciales;

b. Aumentar la eficiencia de las operaciones;

c. Mejorar la utilización de los recursos humanos y materiales;

d. Detectar alternativas o posibilidades de aumento de rentabilidad de la empresa;

e. Analizar la precisión de la información suministrada a la gerencia;

f. Mejorar los métodos de control interno;

g. Eliminar el derroche y las deficiencias;

Consideraciones tomadas en cuenta como premisa, bajo la esencia de administrar recursos, señalados por el autor del presente artículo, en su "Enfoque Técnico en la Administración de Riesgos Empresariales (2014)":

Administrar, dentro de un contexto de manejo de
RECURSOS HUMANOS y sobre un soporte de TOMA
DE DECISIÓN, los funcionarios al asumir responsa-
bilidades de gestión (...). Ello orientado y alineado
hacia el futuro, marcando el establecimiento estra-
tegias, planes de acción, así como la comunicación
hacia los grupos de interés. (p. 11)

La figura 1 nos muestra esquemáticamente lo expresado.

\section{Alcance de la Auditoria De Gestión}

Evalúa las prácticas de gestión, sus criterios que han de diseñarse para el caso específico que se presente. No existen procedimientos específicos de realización, generalmente aceptados sin que se basen en el conocimiento y experiencia del evaluador.

La aplicación sobre la gestión debe estar orientada al área examinada, identificando y analizando las causas de las ineficiencias e ineficacias y sus consecuencias.

En la auditoria de gestión depende de las circunstancias particulares de cada entidad, por lo que los programas de trabajo deben desarrollarse en concordancia. 


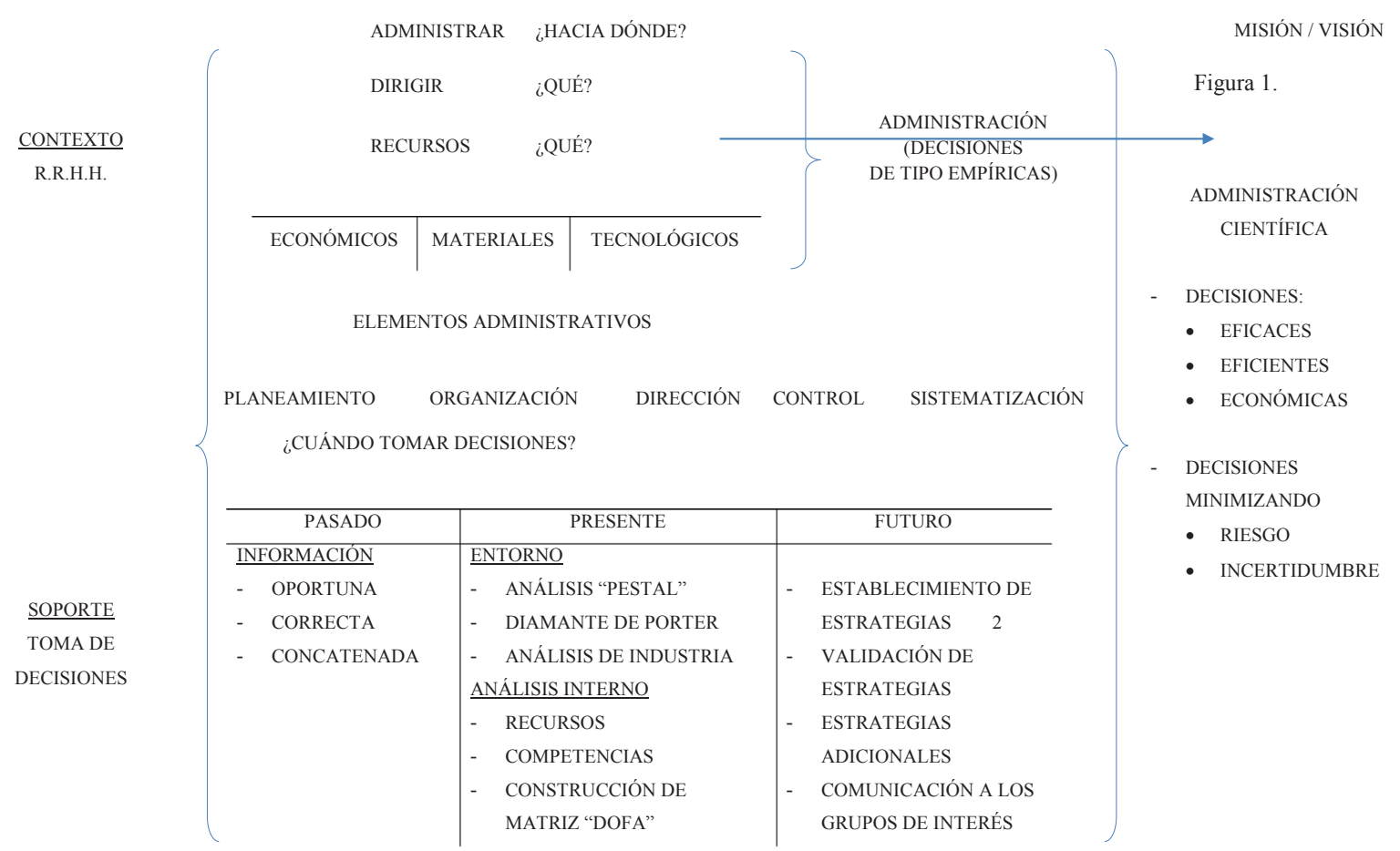

Figura 1. El manejo de los Recursos Humanos y la Toma de Decisiones

Elaboración Propia

Señalaremos algunos enfoques de trabajo, dentro de un plan general, en las áreas principales que conforman la entidad:

1. Auditoría de gestión orientada a la entidad en forma global:

- Evaluación de la posición competitiva

- Evaluación de la estructura organizacional

- Evaluación del clima social

- Evaluación de la dirección estratégica

- Evaluación del cuadro de dirección

2. Auditoria de gestión del área comercial

- Evaluación de las estrategias comerciales

- Análisis de las ofertas de bienes y servicios

- Evaluación de los canales de distribución física

- Evaluación de las políticas de precios

- Evaluación de las funciones de publicidad

- Evaluación de las funciones de ventas
- Evaluación de las promociones de ventas

3. Auditoria de gestión al área de producción

- Diseño y desarrollo del producto

- Productividad técnica y económica

- Diseño del sistema

- Control de calidad

- Almacenaje de inventarios

- Programación de la producción

4. Auditoria de gestión del sistema financiero

- Inversiones

- Capital de trabajo

- Financiamiento a largo plazo

- Planeación financiera

- Evaluación de aspectos internacionales

5. Auditoria de gestión de recursos humanos

- Clima laboral 
- Productividad

- Políticas de selección y productividad

- Políticas de promoción e incentivos

- Diseños de trabajo y puestos de trabajo

6. Auditoria de gestión de sistemas

- Evaluación de los procedimientos administrativos y control interno

- Evaluación de proyectos y programas

- Auditoria de los procesamientos de datos

\section{Normas de Auditoria de Gestión}

Las normas generalmente aceptadas aplicables a los estados financieros por los contadores públicos han sido cuidadosamente desarrolladas por la profesión siendo aprobadas en la "Conferencias Interamericanas de Contabilidad" para regir la auditoría financiera; cabe señalar que hasta ahora no existe normas profesionales oficiales aprobadas para regir la auditoria de gestión, así como las de otros enfoques de trabajo en Auditoria, las que han tomado en base de las normas generalmente aceptadas como plataforma de desarrollo de las practicas a realizarse. Otra alternativa seria elaborar un grupo totalmente diferente de normas profesionales para regir la práctica de auditoría de gestión que no tendría ninguna ventaja especial e incluso confundiría a las personas con poco conocimiento sobre auditoria.

Por ello, se presentan las siguientes normas de auditoria que expresan una adaptación de las normas generalmente aceptadas de tal manera que podrían ser aplicables tanto a la auditoria de gestión como a la auditoría financiera.

1. El examen será efectuado por personas que tienen entrenamiento técnico adecuado y pericia como auditores.

2. En todos los asuntos relacionados con el examen de auditoria la organización de auditoria y los auditores individuales actuarán con integridad y objetividad y mantendrán independencia, no solo en aptitud mental, sino también a la vista de otros.
3. Ejercerá la debida diligencia profesional en la ejecución del examen y preparación del informe.

4. El examen será adecuadamente planeado, supervisado y controlado.

5. Se estudiará y evaluará apropiadamente el control interno vigente como base para determinar el grado de confianza que merece y para determinar el alcance de las pruebas a efectuarse mediante los procedimientos de auditoria.

6. Se documentará y evaluará suficientemente evidencia competente para sustentar una base razonable de los hallazgos, conclusiones, recomendaciones y opiniones del auditor.

7. El informe debe expresar si las practicas gerenciales en las áreas examinadas están de acuerdo con las principios y prácticas gerenciales generalmente aceptadas y si dichas prácticas maximizan la eficiencia, efectividad y economía al orientarse a los objetivos de la entidad.

8. El informe expresara si los principios y prácticas generalmente aceptadas han sido observados consistentemente en las áreas examinadas.

9. El informe comunicará y será limitado a todos los asuntos significativos. Los que deben entenderse como razonablemente adecuados.

10. El informe contendrá los hallazgos, conclusiones, recomendaciones constructivas dirigidas a aumentar le eficiencia, eficacia y economía de la gestión.

Desde el punto personal resaltaremos, el/ los auditor/es como condición previa deberán poseer un entrenamiento técnico adecuado y pericia como tales.

Así mismo, en todos los asuntos relacionados con el examen de auditoría de la organización de auditoría y los auditores individuales 
actuaran con integridad y objetividad, manteniendo la independencia no solo en aptitud mental, sino también a la vista de otros.

\section{La Calidad en la Gestión}

Se encuentra constituida por directrices y objetivos empresariales, relativos a la calidad, difundidos por las direcciones generales; forma parte de la política institucional global y por consiguiente debe ser aprobada por la alta dirección.

Es una función general de la gestión que determina y aplica la política de calidad. Así mismo, incluye la planificación estratégica, la asignación de recursos y otras actividades sistemáticas, tales como la planificación, las operaciones y las evaluaciones relativas a la calidad.

Desde el punto vista de la eficacia, el aseguramiento de la calidad implica, una evaluación permanente de aquellos factores que influyen en la adecuación del manejo de los recursos de la entidad, considerados en los programas pre establecidos de auditoría; tomaremos en cuenta la aplicación en las prácticas de auditoria de gestión las siguientes normas como ejemplo, las de la Organización Internacional de Normalización (ISO), existen muchas, dependiendo del objetivo y enfoque del examen:

- ISO 9000 Como padrón de calidad.

- ISO 9001 Actividades de diseño, desarrollo, producción, y servicio post-venta.

- ISO 9002 Actividades en el aseguramiento de la calidad en la producción y la instalación.

- ISO 9003 Actividades de inspección y los ensayos finales.

- ISO 9004 Da pautas a la Gerencia para el desarrollo de un sistema de calidad.

\section{Metodología de la Auditoria de Gestión}

Esencialmente debe comprender cuatro elementos de trabajo básico:

1. Evaluación del plan de gestión. - Lo que implica tomar conocimiento de asuntos como:
- Productos, mercado, cliente, canales de distribución y alianzas de colaboración;

- de los objetivos, estrategias y riesgos del negocio;

- de los procesos claves del negocio;

- de los principios básicos de un plan de gestión;

- de los principios fundamentales de la administración estratégica;

- del proceso de la administración estratégica;

- de la elaboración programática con la naturaleza, alcance y oportunidad de auditoría de gestión.

Debemos considerar en el contexto de las prácticas diseñadas y programas de trabajo, durante la evaluación del plan de gestión comprende el análisis del ambiente interno (fortalezas y debilidades) y el ambiente externo (oportunidades y amenazas); así como a nivel de dirección organizacional su filosofía, objetivos y metas.

Por otro lado, considerar la evaluación del sistema de información documentaria, como los procesos y subprocesos de importancia, identificando los riesgos principales del negocio y si este posee la administración apropiada de los mismos, correlacionados debidamente con el sistema de control interno.

Consideramos que el plan estratégico al realizarse, debe relacionarse con los resultados financieros y operacionales con el fin de identificar desviaciones importantes y tendencias que se presenten; la toma de decisiones correctivas nos permitirá los indicadores de gestión a tomar en cuenta.

2. Diseño de los medidores de desempeño. - Se requiere de un modelo cuantitativo que nos permita llevar cabo fácilmente un seguimiento eficaz; para analizar el desempeño financiero, así 
como el desempeño operacional; se requiere identificar:

- Indicadores que mejor reflejen la metas y objetivos propuestos;

- Indicadores claves del desempeño o factores críticos de éxito;

- Identificar para cada uno de los indicadores propuestos las fuentes de información y la acciones para que estas sean oportunas y confiables.

3. Medición del desempeño. - Es la contratación de lo siguiente:

- Se realizan las mediciones reales con los indicadores seleccionados;

- se compara los resultados reales con los resultados esperados.

4. Elaboración del Informe comunicando los resultados y las recomendaciones. - Es la comunicación de los resultados del examen es la realidad del auditor que va comunicando verbalmente durante el transcurso para obtener el punto de vista de los funcionarios de la entidad y discutir los hallazgos de auditoría con ellos. De igual modo en la conferencia final a los funcionarios principales responsables de la entidad.

El informe final es único documento preparado por el auditor que representa y documenta sus labores de auditoría, el que será entregado a los funcionarios de la empresa, el directorio y otras personas interesadas.

El contenido del informe debe ser redactado en forma narrativa usando los principios de buena redacción de informes. El informe debe ser cuidadosamente revisado para asegurar que no contenga errores mecanográficos u otros que pueden distraer al lector de la importancia del contenido: se propone el siguiente formato para el contenido del informe:
- Síntesis o carta gerencial (opcional)

- Información introductoria (antecedentes muy abreviados)

- Capítulos individuales por cada hallazgo significativos, estructuras: exposición de los hechos, los criterios (principios, normas, políticas, marcos teóricos, etc. con los que contrastamos los hechos incurridos), las conclusiones y las recomendaciones las que deben claramente identificadas las causas que motivaron las consecuencias de los hechos durante la gestión de la entidad.

- Anexos. - (únicamente cuando sean considerados absolutamente necesarios).

5. Implantación de las recomendaciones. - Deben ser revisadas por el personal de línea de la entidad, se familiarizará con los nuevos sistemas y procedimientos, posteriormente responsables de su ejecución.

6. Plan de seguimiento de la implantación. - Se asegura el éxito y se facilita aclaraciones que surjan durante el proceso de innovación. Permite la posibilidad de tratarlos con la entidad.

La labor está incompleta si existen aspectos normativos o legales que no se reconocen o resuelven. El auditor de gestión frecuentemente necesitara asesoría legal en la labor de desarrollo para considerar adecuadamente los aspectos legales.

Así mismo, el auditor de gestión debe brindar a las personas que están o podrían estar adversamente afectados por los informes la oportunidad de hacer acotaciones sobre los comentarios y hallazgos, proporcionar información o explicaciones adicionales antes de presentar el informe, los que deben reconocer y discutirse en el informe hasta un grado apropiado como para hacer una presentación completa y objetiva. 


\section{CONCLUSIÓN}

Muy pocos directivos admitirán la existencia de ineficiencias en sus áreas de responsabilidad, siendo éstas puestas en su conocimiento. El auditor de gestión proporciona esa información. El contenido del presente artículo muestra el enfoque y planteamiento metodológico de cómo se debe llevar a cabo la práctica del examen.

Actualmente por su naturaleza de la labor, la auditoria administrativa, operativa, financiera es realizada por los auditores internos, e independientes cuyos enfoques y alcances se realizan en los niveles operativos y de transacciones ( el antes, el concurrente y el después ), en las actividades de la entidad, aunque no enfocan la gestión y dirección de la empresa desde el punto de vista estratégico funcional de la alta dirección hacia los objetivos/metas de corto, mediano plazo, y largo plazo alineados a la misión y visión pre establecida verificando si éstas cumplen en grados de razonabilidad estimada.

Las firmas especializadas en auditoria cuentan con especialistas en diversas materias - como: ingeniería, economistas, matemáticas, procesamiento automático de datos (PAD), comercialización etc. - lo que permite un caudal de conocimiento y experiencias en esas diversas materias, coadyuvaran en el logro de una eficaz auditoría de gestión, bajo el enfoque propuesto.

La Auditoria de Gestión constituirá un papel que desempeñará cada vez más el auditor del futuro.

\section{REFERENCIAS BIBLIOGRÁFIACAS}

Aj. Audit of Computer Systems-1998. Paraninfo, Madrid, España.

Añi Ugaz, Carlos, 2001, "NORMAS ISO", Editorial Mantaro, Jr. Canta 530, LIMA, Perú.

Contraloría General de la Republica. Normas Técnicas de Control. Lima, Perú.

Dykerman, Francis G. (1969), Financial reporting systems and techniques, Publicheb by: Prentice- Hall.

Espiñán Gaitán Rodrigo. 2006. Control Interno y Fraudes. Ecoe Ediciones, Bogotá, Colombia.

Fernández Arenas, José Antonio, 1966," La auditoría Administrativa", Editorial JUS, S.A., Plaza de Abosolo, Col. Guerrero, México 3 D.F.

Franklin F. Enrique Benjamín. 2001. Auditoria Administrativa. McGraw-HILL Interamericana EditoreS, S.A. México.

Hevia Eduardo, 2012," Manual de Auditoría Interna", Editorial CENTRUM, Paseo de Gracia 26, Barcelona, España.

Mantilla B. Samuel Alberto-2000, Control Interno, Estructura Conceptual Integrada-Committee Of Sponsoring Organizatipons Of The Treadway Commission (COSO), Santa Fe de Bogotá, Colombia.

Pelazas Manso, Ángeles, 2016, “Auditoria de las áreas de la empresa", Editorial Paraninfo, España.

Rivera León, Félix Armando 2016, "Enfoque Técnico en la Administración de Riesgos Empresariales (2014)" Editorial Académica Española, Barcelona España.

Thierauf J. Robert, 1986 "Auditoria Administrativa", Editorial Limusa, Mexico.

William P. Leonard. 1980. Auditoria Administrativa. Editorial Diana. México. 